\title{
A survey on awareness of genetic counseling for non-invasive prenatal testing: the first year experience in Japan
}

\author{
Junko Yotsumoto $^{1,2}$, Akihiko Sekizawa ${ }^{2}$, Nobuhiro Suzumori ${ }^{3}$, Takahiro Yamada ${ }^{4}$, Osamu Samura ${ }^{5}$, \\ Miyuki Nishiyama $^{6}$, Kiyonori Miura ${ }^{7}$, Hideaki Sawai ${ }^{8}$, Jun Murotsuki ${ }^{9}$, Michihiro Kitagawa ${ }^{10}$, \\ Yoshimasa Kamei ${ }^{11}$, Hideaki Masuzaki ${ }^{7}$, Fumiki Hirahara ${ }^{12}$, Toshiaki Endo ${ }^{13}$, Akimune Fukushima ${ }^{14}$, \\ Akira Namba $^{11}$, Hisao Osada ${ }^{15}$, Yasuyo Kasai ${ }^{16}$, Atsushi Watanabe ${ }^{17}$, Yukiko Katagiri ${ }^{18}$, Naoki Takeshita ${ }^{18}$, \\ Masaki Ogawa ${ }^{19}$, Takashi Okai ${ }^{20}$, Shunichiro Izumi ${ }^{21}$, Haruka Hamanoue ${ }^{12}$, Mayuko Inuzuka ${ }^{1}$, \\ Kazufumi Haino ${ }^{22}$, Naoki Hamajima ${ }^{23}$, Haruki Nishizawa ${ }^{24}$, Yoko Okamoto ${ }^{25}$, Hiroaki Nakamura ${ }^{26}$, \\ Takeshi Kanegawa $^{27}$, Jun Yoshimatsu ${ }^{28}$, Shinya Tairaku ${ }^{29}$, Katsuhiko Naruse ${ }^{30}$, Hisashi Masuyama ${ }^{31}$, \\ Maki Hyodo ${ }^{32}$, Takashi Kaji ${ }^{33}$, Kazuhisa Maeda ${ }^{34}$, Keiichi Matsubara ${ }^{35}$, Masanobu Ogawa ${ }^{36}$, \\ Toshiyuki Yoshizato $^{37}$, Takashi Ohba ${ }^{38}$, Yukie Kawano ${ }^{39}$, Haruhiko Sago ${ }^{6}$ and Japan NIPT Consortium ${ }^{40}$
}

The purpose of this study is to summarize the results from a survey on awareness of genetic counseling for pregnant women who wish to receive non-invasive prenatal testing (NIPT) in Japan. As a component of a clinical study by the Japan NIPT Consortium, genetic counseling was conducted for women who wished to receive NIPT, and a questionnaire concerning both NIPT and genetic counseling was given twice: once after pre-test counseling and again when test results were reported. The responses of 7292 women were analyzed. They expressed high satisfaction with the genetic counseling system of the NIPT Consortium (94\%). The number of respondents who indicated that genetic counseling is necessary for NIPT increased over time. Furthermore, they highly valued genetic counseling provided by skilled clinicians, such as clinical geneticists or genetic counselors. The vast majority $(90 \%)$ responded that there was sufficient opportunity to consider the test ahead of time. Meanwhile, women who received positive test results had a poor opinion and expressed a low-degree satisfaction. We confirmed that the pre-test genetic counseling that we conducted creates an opportunity for pregnant women to sufficiently consider prenatal testing, promotes its understanding and has possibilities to effectively facilitate informed decision making after adequate consideration. A more careful and thorough approach is considered to be necessary for women who received positive test results.

Journal of Human Genetics (2016) 61, 995-1001; doi:10.1038/jhg.2016.96; published online 8 September 2016

\footnotetext{
${ }^{1}$ Natural Science Division, Faculty of Core Research, Ochanomizu University, Tokyo, Japan; ${ }^{2}$ Department of Obstetrics and Gynecology, Showa University School of Medicine, Tokyo, Japan; ${ }^{3}$ Division of Clinical and Molecular Genetics, Department of Obstetrics and Gynecology, Nagoya City University Graduate School of Medical Sciences, Nagoya, Japan; ${ }^{4}$ Department of Obstetrics and Gynecology, Hokkaido University Graduate School of Medicine, Sapporo, Japan; ${ }^{5}$ Department of Obstetrics and Gynecology, The Jikei University School of Medicine, Tokyo, Japan; ${ }^{6} \mathrm{Center}$ of Maternal-Fetal, Neonatal and Reproductive Medicine, National Center for Child Health and Development, Tokyo, Japan; ${ }^{7}$ Department of Obstetrics and Gynecology, Nagasaki University Graduate School of Biomedical Sciences, Nagasaki, Japan; ${ }^{8}$ Department of Obstetrics and Gynecology, Hyogo College of Medicine, Nishinomiya, Japan; ${ }^{9}$ Department of Maternal and Fetal Medicine, Tohoku University Graduate School of Medicine, Miyagi-Children's Hospital, Sendai, Japan; ${ }^{10}$ Department of Obstetrics and Gynecology, Sanno Hospital, Tokyo, Japan; ${ }^{11}$ Department of Obstetrics and Gynecology, Saitama Medical University Hospital, Saitama, Japan; ${ }^{12}$ Department of Obstetrics and Gynecology, Yokohama City University Graduate School of Medicine, Yokohama, Japan; ${ }^{13}$ Department of Obstetrics and Gynecology, Sapporo Medical University School of Medicine, Sapporo, Japan; ${ }^{14}$ Department of Clinical Genetics, Iwate Medical University School of Medicine, Morioka, Japan; ${ }^{15}$ Department of Obstetrics and Gynecology, Chiba University Graduate School of Medicine, Chiba, Japan; ${ }^{16}$ Department of Obstetrics and Gynecology, Japanese Red Cross Medical Center, Tokyo, Japan; ${ }^{17}$ Division of Clinical Genetics, Nippon Medical School Hospital, Tokyo, Japan; ${ }^{18}$ Department of Obstetrics and Gynecology, Toho University Omori Medical Center, Tokyo, Japan; ${ }^{19}$ Perinatal Medical Center, Tokyo Women's Medical University Hospital, Tokyo, Japan; ${ }^{20}$ Maternal and Child Health Center, Aiiku Hospital, Tokyo, Japan; ${ }^{21}$ Department of Obstetrics and Gynecology, Tokai University School of Medicine, Isehara, Japan; ${ }^{22}$ Department of Obstetrics and Gynecology, Niigata University Medical and Dental Hospital, Niigata, Japan; ${ }^{23}$ Department of Clinical Genetics, Nagoya City West Medical Center, Nagoya, Japan; ${ }^{24}$ Department of Obstetrics and Gynecology, Fujita Health University, Toyoake, Japan; ${ }^{25}$ Department of Obstetrics, Osaka Medical Center and Research Institute for Maternal and Child Health, Osaka, Japan; ${ }^{26}$ Department of Obstetrics, Osaka City General Hospital, Osaka, Japan; ${ }^{27}$ Department of Obstetrics and Gynecology, Osaka University Faculty of Medicine, Osaka, Japan; ${ }^{28}$ Department of Perinatology and Gynecology, National Cerebral and Cardiovascular Center, Suita, Japan; ${ }^{29}$ Department of Obstetrics and Gynecology, Kobe University Graduate School of Medicine, Kobe, Japan; ${ }^{30}$ Department of Obstetrics and Gynecology, Nara Medical University, Kashihara, Japan; ${ }^{31}$ Okayama University Graduate School of Medicine, Okayama, Japan; ${ }^{32}$ Hiroshima University Graduate School of Medicine, Hiroshima, Japan; ${ }^{33}$ The University of Tokushima Faculty of Medicine, Tokushima, Japan; ${ }^{34}$ Department of Obstetrics and Gynecology, Shikoku Medical Center for Children and Adults, Zentuji, Japan; ${ }^{35}$ Department of Obstetrics and Gynecology, Ehime University School of Medicine, Toon, Japan; ${ }^{36}$ Department of Obstetrics and Gynecology, Clinical Research Institute, National Hospital Organization Kyushu Medical Center, Fukuoka, Japan; ${ }^{37}$ Center for Maternal, Fetal and Neonatal Medicine, Fukuoka University Hospital, Fukuoka, Japan; ${ }^{38}$ Department of Obstetrics and Gynecology, Kumamoto University, Kumamoto, Japan and ${ }^{39}$ Genetic Counselling Office, Oita University Hospital, Oita, Japan

${ }^{40}$ Members of Japan NIPT Consortium are listed before references.

Correspondence: Dr J Yotsumoto, Natural Science Division, Faculty of Core Research, Ochanomizu University, 2-1-1 Otsuka, Bukyo-ku, Tokyo 112-8610, Japan.

E-mail: yotsumoto.junko@ocha.ac.jp

Received 18 November 2015; revised 1 July 2016; accepted 3 July 2016; published online 8 September 2016
} 


\section{INTRODUCTION}

Prenatal genetic counseling is valuable from the perspective of respecting the individual woman's autonomy regarding pregnancy and childbirth, as well as from the perspective of various ethical aspects. ${ }^{1-3}$ Traditional invasive prenatal diagnostic methods such as amniocentesis or chorionic villus sampling involve a small but definite risk of pregnancy loss; ${ }^{4,5}$ thus, it is mandatory to obtain informed consent before these procedures are performed. In contrast, non-invasive prenatal testing for fetal aneuploidy from cell-free DNA in maternal plasma (non-invasive prenatal testing (NIPT)) involves no risk of pregnancy loss from the test itself, and no medical disadvantages that offset the advantages of the test. ${ }^{6}$ However, due to its convenience, there is a great concern that the test may be offered without sufficient genetic counseling and informed consent before testing, and it is very questionable whether sufficient explanation will be given prior to the test to ensure the individual woman's autonomy and the ethics. ${ }^{7-9}$ It would be extremely difficult to reserve an adequate amount of time to explain NIPT in today's busy perinatal clinical care facilities. Moreover, there is also a concern that its high precision could be overvalued, which could lead to an increased number of cases that are diagnosed based on NIPT results. ${ }^{10}$

In 2011, we conducted a survey on the clinical application of NIPT, with the objectives of elucidating the level of awareness of NIPT among genetic medical professionals and pregnant women and identifying an appropriate way to conduct NIPT. The results of this survey showed that pregnant women have high expectations regarding NIPT and that they do not consider counseling to be important. ${ }^{11}$

The goal of genetic counseling for pregnant women who undergo NIPT is to provide them with adequate information about NIPT on such aspects as the accuracy of the test, the procedure, limitations, target diseases and options depending on the result to facilitate an informed and autonomous decision on whether or not they should proceed with testing. Clinicians also need to ensure that pregnant women are adequately counseled to make autonomous and informed decisions regarding NIPT and to ensure that they understand the results and limitations of the test. ${ }^{3,12}$

Based on the existing genetic counseling model of prenatal testing, various statements and guidelines have been published regarding the clinical use of NIPT. ${ }^{13,14}$ These recommendations uniformly require pre-test counseling by a genetic counselor or another skilled individual, and stipulate that genetic counseling should be available to any pregnant woman who receives a positive result. However, the effects of genetic counseling remain unknown. Thus, the Japan NIPT Consortium was formed in July 2012 to introduce the test along with adequate genetic counseling. The Japan NIPT Consortium planned to initiate NIPT in Japan as a clinical study. ${ }^{15}$ We herein report and summarize the 1-year results from a survey on awareness of genetic counseling.

\section{MATERIALS AND METHODS}

Pregnant women who are identified as being at an increased risk of chromosomal disorders due to advanced maternal age, the results of ultrasound marker or maternal serum marker tests, or a family history of chromosomal abnormalities or those who have a high risk of being a translocation carrier were eligible for NIPT. The present clinical study was conducted in 34 centers that met the facility conditions of the Japan Association of Medical Sciences and which were certified by the association.

Questionnaires were given to pregnant women who received genetic counseling from April 2013 to March 2014. Genetic counseling was conducted by a clinical geneticist or genetic counselor within a specialized outpatient unit. The counseling session was designed to be over $30 \mathrm{~min}$ in duration. At the counseling sessions, the clinical geneticist or counselor used common counseling materials (created by the NIPT consortium), which included
80 pages of material with numerous graphs and tables, which explained congenital disease and prenatal testing in a straightforward manner. After the initial counseling session, the women were also given a leaflet which summarized the content of the counseling. The explanation of test results was also the responsibility of the clinical geneticist or genetic counselor as a component of the genetic counseling. The questionnaire was created based on the results of a survey that we conducted in 2012, and which have been reported elsewhere. ${ }^{11}$ In the present study, the questionnaires were given to each of the pregnant women who received genetic counseling in relation to prenatal genetic testing, both before NIPT and after the results of the test were explained. In the questionnaires, we used a five-choice method, which included the following options: 'I strongly agree', 'I somewhat agree', 'Neither agree not disagree', 'I somewhat disagree' and 'I strongly disagree'. The questionnaires, which were anonymous and self-administered, were recovered at each center. They were subsequently mailed to a third-party NIPT data center for data entry. Each of the participating centers obtained approval for this clinical study from their respective ethics committees and written informed consent was obtained from each of the subjects.

SPSS version 20 (IBM Corporation, Armonk, NY, USA) was used for the statistical analysis. For assessment, each item was weighted based on women's choices; a non-parametric test was used in the analyses of the data from the five-choice answers. The Mann-Whitney U-test (two groups) and KruskalWallis test (three or more groups) were used to analyze the ranked responses, the rank correlation coefficient test was used to determine rank correlation, and the $X^{2}$-test was used to analyze nominal responses.

\section{RESULTS}

Survey questionnaires were recovered from 8067 pregnant women who received genetic counseling in relation to prenatal genetic testing, including NIPT. NIPT was performed in 7740 cases in 1 year. ${ }^{15}$ We analyzed 7292 questionnaires that had been completed by women before and after NIPT.

\section{Patient background}

The characteristics of the women who received genetic counseling are shown in Table 1. Their mean age was 38.3 years (range: $21-49$ years). The initial counseling session most commonly took place at 12-13 gestational weeks. In regard to the education of the women, $42.9 \%$ were university graduates and $6.2 \%$ had graduated from graduate school. The majority of the women had an annual household income of $\geqslant 7$ million yen.

In most cases, the indication for NIPT (multiple answers allowed) was advanced maternal age (96.5\%). In addition, $14.4 \%$ of the women underwent NIPT based on a recommendation from their family.

\section{Comprehension of NIPT}

In the questionnaires after initial genetic counseling, the women were asked to indicate whether they were able to understand the characteristic features of NIPT using 5-point scale answers. The results showed that the women understood that NIPT involved no risk of pregnancy loss, that amniocentesis is necessary when test results are positive, the indications for NIPT and the targeted chromosomal abnormalities, ranked in order of highest understanding. Combining 'I strongly agree' and 'I somewhat agree', $\geqslant 90 \%$ of the women understood each of the evaluation items. It was also revealed that there was a high understanding that the test 'does not lead to a definitive diagnosis.' (Figure 1).

\section{The evaluation of NIPT}

The women were asked to indicate which of the aspects of NIPT they valued, using the 5-point scale. The most highly rated responses, ranked in order, were as follows: 'no risk of pregnancy loss', 'necessity of genetic counseling' and 'availability of testing during early 
Table 1 The characteristics of the pregnant women who underwent genetic counseling $(n=7292)$

\begin{tabular}{|c|c|}
\hline & Number (\%) \\
\hline \multicolumn{2}{|l|}{ Maternal age (years) } \\
\hline$\leqslant 34$ & $347(4.8 \%)$ \\
\hline 35-39 & $4548(62.4 \%)$ \\
\hline $40-44$ & $2333(32.0 \%)$ \\
\hline $45-49$ & $59(0.8 \%)$ \\
\hline NA & $5(0.1 \%)$ \\
\hline \multicolumn{2}{|c|}{ Gestational week at first genetic counseling session (weeks of gestation) } \\
\hline-9 & $38(0.5 \%)$ \\
\hline $10-11$ & $1416(19.4 \%)$ \\
\hline $12-13$ & $3056(41.9 \%)$ \\
\hline $14-15$ & $2238(30.7 \%)$ \\
\hline $16-17$ & $466(6.4 \%)$ \\
\hline $18-$ & $38(0.5 \%)$ \\
\hline NA & $40(0.5 \%)$ \\
\hline \multicolumn{2}{|l|}{ Parity } \\
\hline 0 & $3776(51.8 \%)$ \\
\hline 1 & $2418(33.2 \%)$ \\
\hline$\geqslant 2$ & $820(11.2 \%)$ \\
\hline NA & $278(3.8 \%)$ \\
\hline \multicolumn{2}{|l|}{ Fertility treatment } \\
\hline No & $4202(57.6 \%)$ \\
\hline Yes & $3079(42.2 \%)$ \\
\hline Multiple answers & (3475) \\
\hline Timing & $843(27.3 \%)$ \\
\hline Artificial insemination & $311(10.1 \%)$ \\
\hline IVF & $1038(33.6 \%)$ \\
\hline ICSI & $933(30.2 \%)$ \\
\hline Others & $350(11.3 \%)$ \\
\hline NA & $11(1.5 \%)$ \\
\hline \multicolumn{2}{|l|}{ Education } \\
\hline High school & $1359(18.6 \%)$ \\
\hline Junior college & $2326(31.9 \%)$ \\
\hline University & $3127(42.9 \%)$ \\
\hline Graduate school & $453(6.2 \%)$ \\
\hline NA & $128(1.8 \%)$ \\
\hline \multicolumn{2}{|l|}{ Total family income $(\times \$ 100)$} \\
\hline-415 & $1192(16.3 \%)$ \\
\hline $416-580$ & $1916(26.3 \%)$ \\
\hline $581-830$ & $2040(28.0 \%)$ \\
\hline $831-1250$ & $1294(17.7 \%)$ \\
\hline $1251-$ & $722(9.9 \%)$ \\
\hline NA & $128(1.8 \%)$ \\
\hline \multicolumn{2}{|c|}{ Indication of NIPT (multiple answers) } \\
\hline Maternal age & $7021(96.5 \%)$ \\
\hline Ultrasonography & $145(2.0 \%)$ \\
\hline Serum marker & $44(0.6 \%)$ \\
\hline Family history (own child) & $159(2.2 \%)$ \\
\hline Family history (relative) & $206(2.2 \%)$ \\
\hline Recommendation of the family & $1051(14.4 \%)$ \\
\hline Recommendation of the doctor & $168(2.3 \%)$ \\
\hline Others & $563(7.7 \%)$ \\
\hline
\end{tabular}

Abbreviations: ICSI, intracytoplasmic sperm injection; IVF, in vitro fertilization; NA, not applicable; NIPT, non-invasive prenatal testing. pregnancy' (Figure 2). In contrast, there were very few women who responded that 'women with low risk of chromosomal abnormalities should also be able to receive NIPT.' In addition, $\geqslant 85 \%$ of the women valued specific features of NIPT, such as 'no risk of pregnancy loss' and 'safety for both mother and fetus', 'availability of testing during early pregnancy', and 'high diagnostic accuracy'. Furthermore, the rank sum analysis revealed that the rating of 'genetic counseling is necessary' improved in latter half compared with the first half. It is considered that evaluation of genetic counseling in each woman might have been improved according to the experience of the genetic counseling on prenatal testing (up to September 2013) (Table 2).

\section{The evaluation of genetic counseling}

The women were asked to assess and indicate their impressions of genetic counseling using a similar 5-point scale. The results showed that a large number of the women indicated positive responses to 'I was glad I received counseling from a clinician who is a genetics specialist', 'genetic counseling is necessary for NIPT' and 'a clinician who is skilled in genetic counseling should conduct this test'. Moreover, $92 \%$ of the women responded that they were pleased that they received counseling from the specialists. Furthermore, 91\% of the women responded 'genetic counseling is necessary for this test' and $80 \%$ responded 'I was able to thoroughly consider the ethical aspects.'

In regard to the necessity of genetic counseling, $91 \%$ of the women had a positive response. Moreover, this tendency was greater after NIPT than before NIPT. There was a trend for greater knowledge of genetic counseling with greater parity and a higher level of education.

Furthermore, regarding the survey that was conducted after genetic counseling in relation to the test results, $>90 \%$ of the women gave a positive response and only $3 \%$ gave a negative response to the question, 'Was genetic counseling a sufficient opportunity to consider prenatal testing?' (Figure 3).

\section{Women with positive NIPT results vs women with negative NIPT results}

Comparing the ratings of NIPT by women who had positive results with those of women who had negative results, we found that the women with negative results indicated a greater level of satisfaction with NIPT. Specifically, those with negative test results indicated that 'High diagnostic accuracy 'more people should know about the test', 'women with a low risk of chromosomal abnormalities should also be able to receive NIPT' and that 'No risk of pregnancy loss' (Table 3). Moreover, the $96.5 \%(6582 / 6820)$ of the women who had negative test results indicated that they would choose to undergo NIPT in their next pregnancy; this percentage was significantly higher than that in women with positive test results $(87.3 \%, 227 / 260 ; P<0.0001)$. Conversely, those with positive test results did not view the test to be highly precise or safe, and had a strong tendency to opt for amniocentesis in their next pregnancy. Moreover, it is estimated that anxiety tended to be persistent, and the women tended to want further explanation over a longer duration of time in comparison to the subjects with negative test results (Table 4).

\section{The correlation between the duration of genetic counseling and} patient satisfaction

Although genetic counseling is designed to be over $30 \mathrm{~min}$ in duration, the patient-reported durations indicated that $4.5 \%$ of the counseling sessions were $<10 \mathrm{~min}$ in length, and that $23.6 \%$ were $10-20 \mathrm{~min}$ in length. The duration of counseling was divided into subgroups by 10 -min intervals, and satisfaction regarding the amount of 


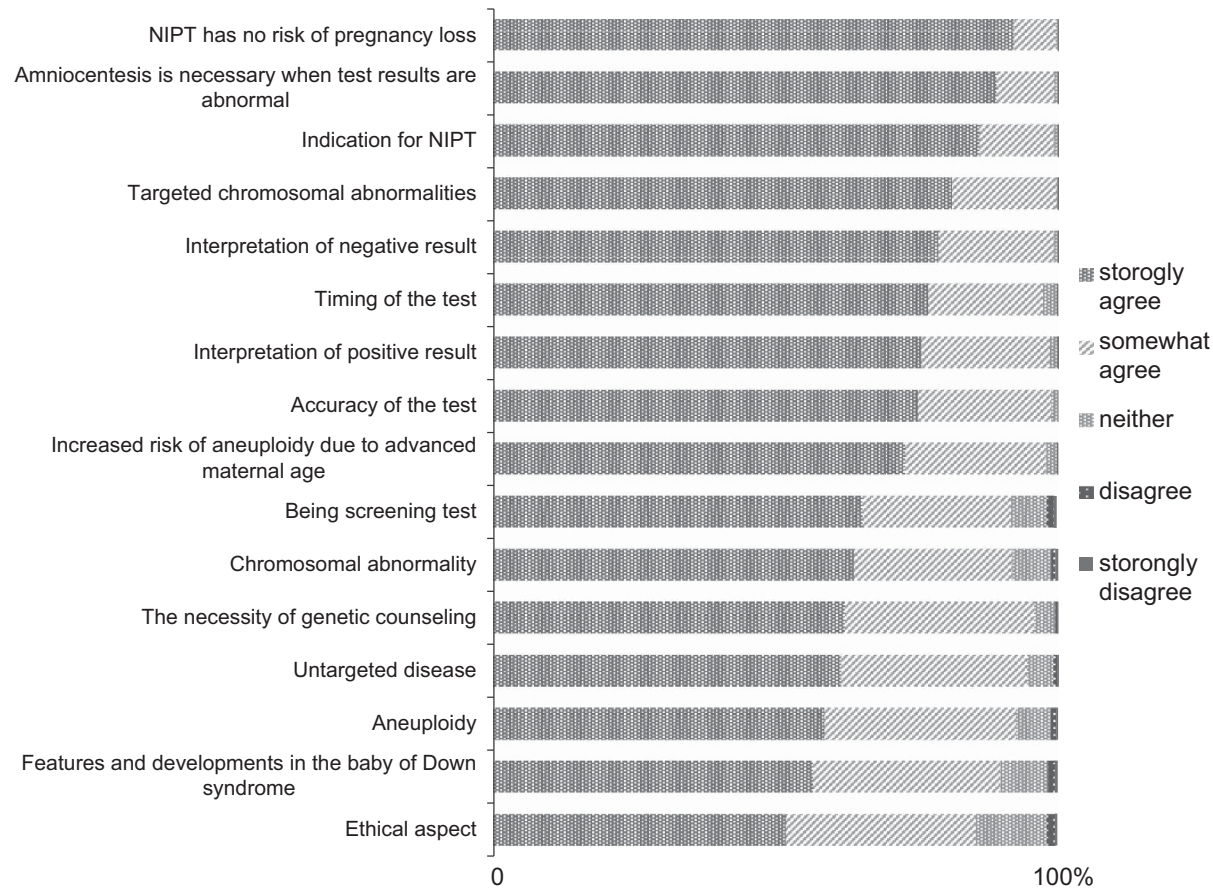

Figure 1 The comprehension with regard to NIPT $(n=7292)$. NIPT, non-invasive prenatal testing. A full color version of this figure is available at the Journal of Human Genetics journal online.

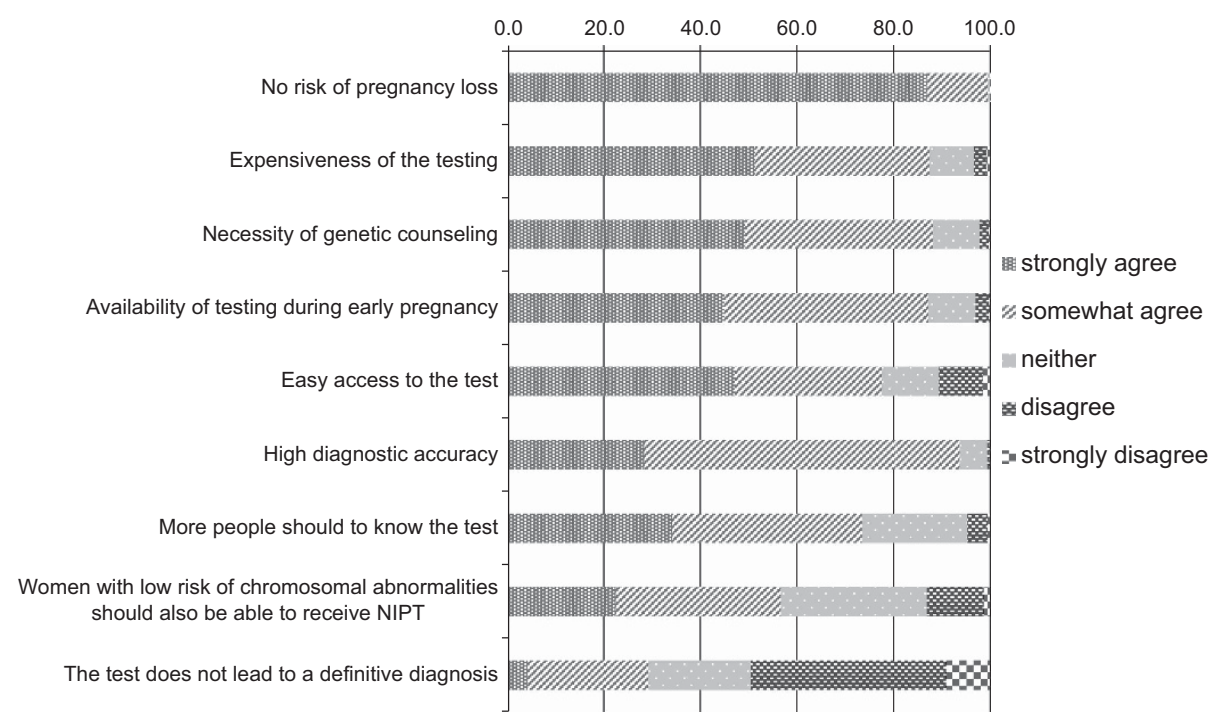

Figure 2 The evaluation of NIPT $(n=7292)$. NIPT, non-invasive prenatal testing. A full color version of this figure is available at the Journal of Human Genetics journal online.

information and the ratings of the necessity of genetic counseling were compared among the groups. The results showed that patient satisfaction improved in genetic counseling sessions of 10-20 min in duration in comparison to sessions of $<10 \mathrm{~min}$. Patient satisfaction remained nearly constant beyond 20 min: we could not observe any difference among the sessions that were $10-20 \mathrm{~min}, 20-30 \mathrm{~min}$ or $\geqslant 30 \mathrm{~min}$ in duration (Figure 4).

\section{DISCUSSION}

In Japan, NIPT was initiated through the present nationwide clinical study. ${ }^{15}$ One of the notable characteristics of the women who received genetic counseling in relation to NIPT is that the mean age of the women at the initial counseling session was 38.3 years. Advanced maternal age was the reason why $96 \%$ of the women in this study underwent NIPT. An extremely low number of women opted to 
Table 2 The evaluation of NIPT

\begin{tabular}{|c|c|c|c|}
\hline \multirow[b]{2}{*}{ Evaluation item } & \multicolumn{2}{|c|}{ Averaged rank ${ }^{a}$} & \multirow[b]{2}{*}{$\mathrm{P}$-value ${ }^{\mathrm{b}}$} \\
\hline & $\begin{array}{l}\text { First half } \\
(\mathrm{N}=2911)\end{array}$ & $\begin{array}{l}\text { The latter half } \\
\qquad(\mathrm{N}=3230)\end{array}$ & \\
\hline No risk of pregnancy loss & 7.22 & 7.20 & 0.40 \\
\hline Expensiveness of the testing & 5.64 & 5.71 & 0.99 \\
\hline Necessity of genetic counseling & 5.55 & 5.62 & 0.44 \\
\hline $\begin{array}{l}\text { Availability of testing during early } \\
\text { pregnancy }\end{array}$ & 5.63 & 5.45 & 0.00 \\
\hline Easy access to the test & 5.14 & 5.22 & 0.15 \\
\hline High diagnostic accuracy & 4.93 & 5.03 & 0.18 \\
\hline More people should know about the test & 4.76 & 4.73 & 0.36 \\
\hline $\begin{array}{l}\text { Women with a low risk of chromosomal } \\
\text { abnormalities should also be able to } \\
\text { receive NIPT }\end{array}$ & 3.87 & 3.79 & 0.02 \\
\hline $\begin{array}{l}\text { The test does not lead to a definitive } \\
\text { diagnosis }\end{array}$ & 2.26 & 2.25 & 0.76 \\
\hline
\end{tabular}

Abbreviation: NIPT, non-invasive prenatal testing.

aFriedman test: in each group of first half and second half, a rank sum analysis was performed.

The Friedman test (non-parametric statistical test) is used to detect differences in paired

multiple items and indicates by the rank sum result.

bMann-Whitney test: the difference between first half and second half was analyzed.

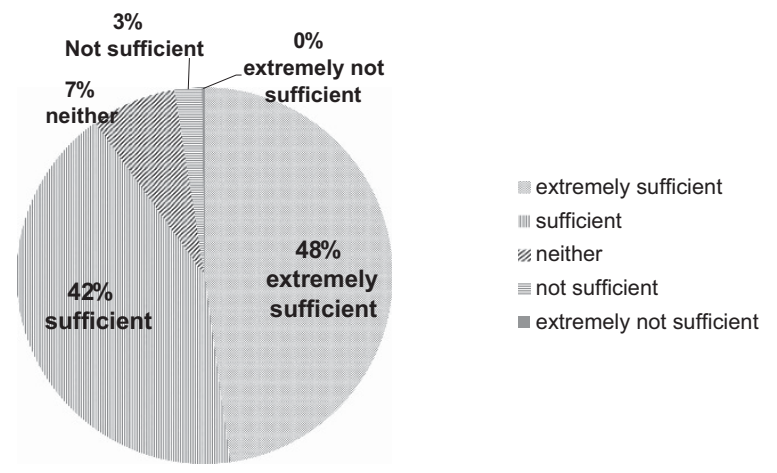

Figure 3 Was genetic counseling a sufficient opportunity to think about prenatal testing? ( $n=7292)$. A full color version of this figure is available at the Journal of Human Genetics journal online. undergo NIPT after a serum marker test or an ultrasound marker test. This reflects the situation in Japan, where such tests are not generally conducted for the purpose of screening. The patients in the present study clearly had a higher education level; about half (49.1\%) of the pregnant women in the present study were university graduates or more, the university enrollment rate was $22.9 \%$ in 1995 among the general population, when the pregnant women of this study would have been attending university. More than half of the participants had an annual household income of $\geqslant 7$ million yen. In 2010, the mean annual household income in Japan was 5.5 million yen and the median annual household income was 4.4 million yen. These findings suggest that women in higher-income brackets are more likely to undergo NIPT. This is influenced by the fact that in Japan, NIPT involves an out-of-pocket expense of 180000-260000 yen (\$1500-\$2270).

In regard to the comprehension of NIPT at genetic counseling, $\geqslant 90 \%$ of the women responded in self-reported that they could fully understand the content of the explanation (Figure 1). We hypothesize that this occurred because skilled clinicians who are genetics specialists were in charge of the genetic counseling. The use of easy to understand and well-illustrated counseling material was also thought to be effective. However, the explanations on chromosomal abnormalities and Down syndrome, as well as the ethical issues involved in prenatal testing were considered to be relatively difficult to understand. Special schemes such as the preparation of comprehensible explanatory materials and taking time to thoroughly provide explanations were considered to be essential to improve the understanding in relation to these issues.

Moreover, there were very few women who deemed that NIPT is a 'test that anyone can take'.

Regarding the evaluation on genetic counseling, $\geqslant 90 \%$ of the women recognized its necessity through their own experience. In addition, that $\geqslant 80 \%$ of the women responded that they were 'able to think carefully about ethical aspects' suggested that this was an effect of taking the time to conduct genetic counseling, as well the opportunity to rethink the decision to undergo prenatal testing (Figure 3). Moreover, the finding that the clinicians who are skilled in genetics took the time to conduct counseling in this clinical study was thought to lead to the high level of satisfaction with genetic counseling. Furthermore, a significantly greater number of women responded that 'genetic counseling is necessary' in the latter 6 months of testing in comparison to the first 6 months; qualitative improvements in genetic counseling practices were suggested to have

Table 3 The evaluation of NIPT (positive vs negative results)

\begin{tabular}{|c|c|c|c|}
\hline \multirow[b]{2}{*}{ Evaluation items } & \multicolumn{2}{|c|}{ Rank sum (N) } & \multirow[b]{2}{*}{ P-value } \\
\hline & Positive & Negative & \\
\hline High diagnostic accuracy & 3240.957 (269) & $3599.965(6903)$ & 0.001 * \\
\hline The test does not lead to a definitive diagnosis & $3942.637(270)$ & $3561.614(6881)$ & $0.002 *$ \\
\hline Availability of testing during early pregnancy & $3657.783(270)$ & $3587.871(6910)$ & 0.551 \\
\hline No risk of pregnancy loss & $3286.952(273)$ & $3607.678(6917)$ & $0.000 *$ \\
\hline Necessity of genetic counseling & 3467.509 (273) & 3594.323 (6905) & 0.274 \\
\hline Women with a low risk of chromosomal abnormalities should also be able to receive NIPT & $3275.411(271)$ & 3596.645 (6897) & $0.009 *$ \\
\hline More people should know about the test & $3341.386(273)$ & 3601.383 (6909) & $0.031^{*}$ \\
\hline Easy access to the test & $3531.678(273)$ & 3599.577 (6920) & 0.569 \\
\hline Expensiveness of the testing & $3492.888(272)$ & 3596.939 (6913) & 0.369 \\
\hline
\end{tabular}

Abbreviation: NIPT, non-invasive prenatal testing.

Mann-Whitney $U$-test, ${ }^{*} P$-value $<0.05$. 
Averaged rank

\begin{tabular}{|c|c|c|c|}
\hline Evaluation items & Positive $(\mathrm{N}=267)$ & Negative $(\mathrm{N}=6840)$ & $\mathrm{P}$-value $\mathrm{b}^{\mathrm{b}}$ \\
\hline I was glad I received counseling from a specialist of clinical genetics & 3.56 & 3.61 & 0.018 \\
\hline Genetic counseling is necessary for NIPT & 3.51 & 3.60 & 0.011 \\
\hline A specialist in genetic counseling should conduct this & 3.38 & 3.42 & 0.227 \\
\hline More time is needed for genetic counseling & 1.49 & 1.31 & 0.001 \\
\hline
\end{tabular}

aFriedman test: in each group of positive result and negative result, a rank sum analysis was performed. The Friedman test (non-parametric statistical test) is used to detect differences in paired multiple items and indicates by the rank sum result.

bMann-Whitney test: the difference between positive result group and negative result group was analyzed.

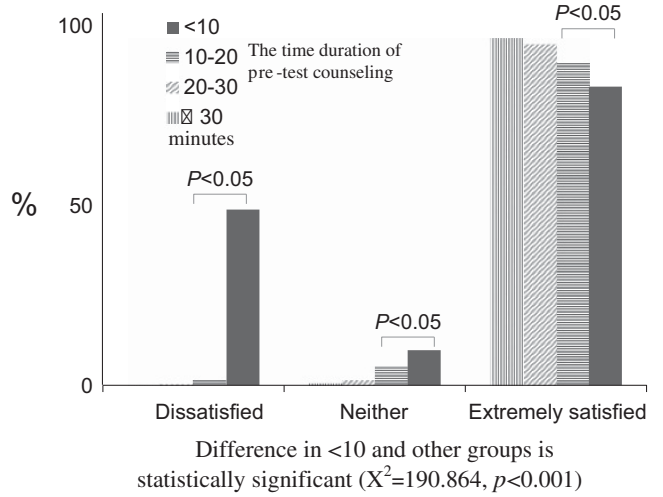

Figure 4 Time and satisfaction with information $(n=7292)$. There was a statistically significant difference in the satisfaction of the $<10$ min group and the other groups $\left(X^{2}=190.864, P<0.001\right)$. A full color version of this figure is available at the Journal of Human Genetics journal online.

contributed to this increase. The findings of the present study indicate that, in the clinical application of NIPT, appropriate genetic counseling is essential, as it improves the patient's understanding of the test and allows her to make an autonomous choice.

The responses to the questions from the perspective of the required time, duration and the quantity of information in genetic counseling in relation to NIPT showed that satisfaction and ratings on the necessity of genetic counseling were greater among respondents who attended counseling sessions that were longer in duration (up to $20 \mathrm{~min}$ ), but that the level of satisfaction remained almost constant beyond $20 \mathrm{~min}$.

A recent study investigated that the average times required for face-to-face genetic counseling for pediatric, oncogenetic, pregnancy with a malformed fetus and pre-amniocentesis counseling sessions were $48 \mathrm{~min}, 37 \mathrm{~min}, 40 \mathrm{~min}$ and $18 \mathrm{~min}$, respectively. ${ }^{16}$ Previous studies have shown that genetic services are labor intensive and extremely time-consuming, due to the time involved in both the face-to-face counseling sessions and indirect patient-related activities. ${ }^{17-20}$ In this current era, there is obviously an increased demand for both face-to-face pre-test counseling (regarding NIPT or other new technologies) and indirect patient-related activities. There is a clearly need for an appropriate genetic service that does not compromise the quality of patient care.

The American Congress of Obstetricians and Gynecologists (ACOG), the National Society of Genetic Counselors (NSGC), the American College of Medical Genetics (ACMG) and the Japanese
Society of Obstetricians and Gynecologists (JSOG) have released guidelines for NIPT. These guidelines share some similarities, 'NIPT is a screening tool', 'pre-test genetic counseling are for all pregnant women', 'post-test counseling are recommended for screenpositive individual' and 'positive NIPT results should be confirmed by CVS/amniocentesis'. . $^{93,21,22}$ We conducted this survey under more strict criteria than were applied in previous studies. ${ }^{15}$ Although the standard and adequate times for pre-test counseling in patients undergoing NIPT are unknown, the level of detail of individual genetic counseling sessions varies and reflects the different policies that are in place in different countries. Genetic counseling represents an opportunity to establish a relationship of trust between patients and medical professionals, and is a chance for pregnant women to receive psycho-educational counseling. Our findings indicate that the preferred duration of genetic counseling sessions for women undergoing NIPT is $\geqslant 20 \mathrm{~min}$, and that the time that is currently allotted for the explanation of NIPT $(\geqslant 30 \mathrm{~min})$ may be suitable.

However, there are individual differences in comprehension, and because a psychosocial approach is also necessary, it is essential to ensure an adequate amount of time to appropriately inform each individual. This indicates that factors other than duration contribute to satisfaction and therefore suggests the need for a qualitative scheme that involves more than simply providing information.

Pregnant women may make various choices after genetic counseling. However, in the present study, we found that a majority of the pregnant women opted for NIPT after undergoing pre-test genetic counseling. Only $8 \%$ of 2041 pregnant women who received pre-test genetic counseling, elected not to undergo NIPT after undergoing counseling. Unfortunately, our data was incomplete, because some institutions in our study group did not collect the questionnaires from the pregnant women who elected not to undergo NIPT after pre-test genetic counseling. The fact that most pregnant women decided to undergo NIPT after pre-test genetic counseling might reflect the characteristics of the pregnant women who wanted to undergo NIPT or it might be affected by the current counseling system for NIPT in Japan. Thus, there is a need to investigate this matter further in the future.

When the NIPT ratings were compared, those who received negative test results reported a significantly higher level of satisfaction with counseling than those who received positive NIPT results. This finding would reflect the anxiety of the test taker after being informed of the positive test result. In addition, dissatisfaction regarding the uncertainty of the test's precision was also evident, especially since the test results and the positive predictive value were explained to the women with positive test results. Moreover, it appears that this led to a stronger tendency for the women to consider amniocentesis in the next pregnancy. These findings demonstrated that the ratings in 
relation to genetic counseling, satisfaction and understanding of NIPT were lower among women with positive test results than among those with negative results. Even with a sufficient advance explanation, there was a great impact on women who received positive test results, and a significant long-term emotional burden was caused by their uncertainty as they waited for a definitive diagnosis. ${ }^{23}$ The required time for genetic counseling was longer among these women; however, since it was considered that women with positive test results may make decisions in a state of reluctance and confusion a more careful approach was considered to be necessary.

Due to the high need for genetic counseling and because it included a large amount of counseling content that requires a particular method of explanation, it is considered necessary that genetic counseling be provided by skilled clinicians in future clinical applications. Moreover, as seen by $90 \%$ of the women who responded that their opportunity to consider the testing was sufficient, the provision of genetic counseling prior to the test provides women with an opportunity to rethink the decision to undergo prenatal testing, and is considered to deter women from the easy acceptance of undergoing the test.

In conclusion, we herein described the 1-year experience in the provision of genetic counseling in relation to the Japanese nationwide NIPT project. We confirmed that the content and methodology of the genetic counseling creates an opportunity for pregnant women to sufficiently consider prenatal testing, and that it promotes the understanding in relation to the test and effectively facilitates the making of informed choices after adequate consideration. ${ }^{24}$ Furthermore, the results revealed that most of women were able to consider the ethical aspects of prenatal testing and the decision to undergo NIPT. On the other hand, it was revealed that women with positive test results had various difficulties regarding genetic counseling in relation to NIPT. Our current study will help to improve the quality of genetic counseling in relation to NIPT, especially in Japan.

\section{CONFLICT OF INTEREST}

The authors declare no conflict of interest.

\section{ACKNOWLEDGEMENTS}

We thank all clinical geneticists and genetic counselors who participated in this study for making this study possible. We thank all the respondents participating in this study. AS and HS were supported by the Grant of National Center for Child Health and Development 24-3, Japan.

\section{STUDY GROUP MEMBERS OF JAPAN NIPT CONSORTIUM}

Rina Akaishi, Takashi Kojima, Yuka Shibata (Hokkaido University Graduate School of Medicine), Seiji Wada, Aiko Sasaki (National Center for Child Health and Development), Nahoko Shirato, Keiko Miyagami, Tatsuko Hirose, Atsuko Saito (Showa University), Tomohiro Tanemoto, Madoka Horiya (The Jikei University), Akinori Miki, Machiko Kimura, Hiroko Nakagami, Taki Kamigaki (Saitama Medical University Hospital), Yuri Hasegawa, Shoko Miura, Noriko Sasaki (Nagasaki University), Mako Ueda, Mariko Ushioda, Chiho Okada, Hiroyuki Tanaka, Mina Morii-Kashima (Hyogo College of Medicine), Naomi Nishikawa (Nagoya City West Medical Center), Kei Hayata (Okayama University Graduate School of Medicine), Hanae Satano (Hiroshima University), Michiko Watanabe, Kaori Arima (Japanese Red Cross Medical Center), Kyoko Kumagai, Eri Takeda, Kumiko Oseto (Nagoya City University), Wakana Abe (Oita University Hospital), Motoko Sasaki (Nippon Medical School Hospital), Tomoo Hirabuki (Odawara City Hospital), Haruya Saji (Fujisawa City Hospital), Hiromi Nagase, Akihito Mochizuki, Hiroshi Ishikawa
(Kanagawa Children's Medical Center), Kimiko Enomoto (Yokohama City University Medical Center), Kaori Sawai (Yokohama Municipal Citizen's Hospital), Rie Suzuki (National Hospital Organization Yokohama Medical Center), Yoshinobu Sugo, Mari Shinoda, (Yokohama City University Hospital), Miki Tanoshita, Kauzmi Takahashi (Tokai University School of Medicine), Yuko Ohnuki, Hiromi Moriya, Naoko Harada, Hiroe Onaka, Ryoko Hikima, Akinobu Kuroki, Norimasa Sawaguchi (Tokai University Hospital).

1 Chervenak, F. A. \& McCullough, L. B. Ethical issues in perinatal genetics. Semin. Fetal Neonatal. Med. 16, 70-73 (2011).

2 Hertig, S. G., Cavalli, S., Burton-Jeangros, C. \& Elger, B. S. 'Doctor, what would you do in my position?' Health professionals and the decision-making process in pregnancy monitoring. J. Med. Ethics 40, 310-314 (2014).

3 Godino, L. et al. Attitudes of women of advanced maternal age undergoing invasive prenatal diagnosis and the impact of genetic counselling. Eur. J. Hum. Genet. 24, 331-337 (2015).

4 Tabor, A. \& Alfirevic, Z. Update on procedure-related risks for prenatal diagnosis techniques. Fetal Diagn. Ther. 27, 1-7 (2010).

5 Akolekar, R., Beta, J., Picciarelli, G., Ogilvie, C. \& D'Antonio, F. Procedure-related risk of miscarriage following amniocentesis and chorionic villus sampling: a systematic review and meta-analysis. Ultrasound Obstet. Gynecol. 45, 16-26 (2015).

6 Tiller, G. E., Kershberg, H. B., Goff, J., Coffeen, C., Liao, W. \& Sehnert, A. J. Women's views and the impact of noninvasive prenatal testing on procedures in a managed care setting. Prenat. Diagn. 35, 428-433 (2014).

7 Alexander, E., Kelly, S. \& Kerzin-Storrar, L. Non-invasive prenatal testing: UK genetic counselors' experiences and perspectives. J. Genet. Couns. 24, 300-311 (2014).

8 Benn, P., Chapman, A. R., Erickson, K., Defrancesco, M. S., Wilkins-Haug, L., Egan, J. F. et al. Obstetricians and gynecologists' practice and opinions of expanded carrier testing and noninvasive prenatal testing. Prenat. Diagn. 34, 145-152 (2014).

9 Buchanan, A., Sachs, A., Toler, T. \& Tsipis, J. NIPT: current utilization and implications for the future of prenatal genetic counseling. Prenat. Diagn. 34, 850-857 (2014).

10 Mennuti, M. T., Cherry, A. M., Morrissette, J. J. \& Dugoff, L. Is it time to sound an alarm about false-positive cell-free DNA testing for fetal aneuploidy? Am. J. Obstet. Gynecol. 209, 415-419 (2013).

11 Yotsumoto, J., Sekizawa, A., Koide, K., Purwosunu, Y., Ichizuka, K., Matsuoka, R. et al Attitudes toward non-invasive prenatal diagnosis among pregnant women and health professionals in Japan. Prenat. Diagn. 32, 674-679 (2012).

12 Wax, J. R., Cartin, A., Chard, R., Lucas, F. L. \& Pinette, M. G. Noninvasive prenatal testing: Impact on genetic counseling, invasive prenatal diagnosis, and trisomy 21 detection. J. Clin. Ultrasound 43, 1-6 (2014).

13 American College of Obstetricians and Gynecologists Committee on Genetics. Committee Opinion No. 545: noninvasive prenatal testing for fetal aneuploidy. Obstet Gynecol 120, 1532-1534 (2012)

14 Wilson, K. L., Czerwinski, J. L., Hoskovec, J. M., Noblin, S. J., Harbison, A., Singletary, C. N. et al. NSGC practice guideline: prenatal screening and diagnostic testing options for chromosome aneuploidy. J. Genet. Couns. 22, 4-15 (2013).

15 Sago, H., Sekizawa, A. \& Japan NIPT consortium. Nationwide demonstration project of next-generation sequencing of cell-free DNA in maternal plasma in Japan: one-year experience. Prenat. Diagn. 35, 331-336 (2014).

16 Sukenik-Halevy, R., Ludman, M. D., Ben-Shachar, S. \& Raas-Rothschild, A. The time-consuming demands of the practice of medical genetics in the era of advanced genomic testing. Genet. Med. 18, 372-377 (2016).

17 Bernhardt, B. A., Tumpson, J. E. \& Pyeritz, R. E. The economics of clinical genetics services. IV. Financial impact of outpatient genetic services on an academic institution. Am. J. Hum. Genet. 50, 84-91 (1992).

18 Pletcher, B. A., Jewett, E. A., Cull, W. L., Brotherton, S. E. \& Hoyme, H. E. The practice of clinical genetics: a survey of practitioners. Genet. Med. 4, 142-149 (2002).

19 Cooksey, J. A., Forte, G., Benkendorf, J. \& Blitzer, M. G. The state of the medical geneticist workforce: findings of the 2003 survey of American Board of Medical Genetics certified geneticists. Genet. Med. 7, 439-443 (2005).

20 McPherson, E., Zaleski, C., Benishek, K., Reynolds, K. \& Rasmussen, K. Clinical genetics provider real-time workflow study. Genet. Med. 10, 699-706 (2008).

21 Gregg, A. R., Gross, S. J., Best, R. G., Thompson, B. H. \& Watson, M. S. ACMG statement on noninvasive prenatal screening for fetal aneuploidy. Genet. Med. 15, 395-398 (2013).

22 National Society of Genetic Counselors. Noninvasive Prenatal Testing/noninvasive Prenatal Diagnosis (NIPT/NIPD). Available at http://nsgc.org/p/bl/et/blogid $=47 \&$ blogaid $=33$ (2012).

23 Weinans, M. J., Huijssoon, A. M., Tymstra, T., Gerrits, M. C., Beekhuis, J. R. \& Mantingh, A. How women deal with the results of serum screening for Down syndrome in the second trimester of pregnancy. Prenat. Diagn. 20, 705-708 (2000).

24 Farrelly, E., Cho, M. K., Erby, L., Roter, D., Stenzel, A. \& Ormond, K. Genetic counseling for prenatal testing: where is the discussion about disability? J. Genet. Couns. 21, 814-824 (2012). 